\title{
Factores relacionados y conductas sobre sexting en estudiantes de enfermería en una universidad pública*
}

Behaviors and related factors about sexting among nursing students in a public college

Fatores relacionados e comportamentos sobre sexting em discentes de enfermagem em uma universidade pública

Marco Esteban Morales Rojas ${ }^{\text {a }}$

Universidad Autónoma de Yucatán, México

marco.morales@correo.uady.mx

ORCID: https://orcid.org/0000-0003-3416-0806

Martha Ofelia Valle Solis

Universidad Autónoma de Nayarit, México

ORCID: https://orcid.org/0000-0001-8772-6549

Verónica Benitez Guerrero

Universidad Autónoma de Nayarit, México

ORCID: https://orcid.org/0000-0002-0036-1784

Juan Fernando López Flores

Hospital Civil de Tepic de los Servicios de Salud de Nayarit,

México

ORCID: https://orcid.org/ 0000-0002-9556-9624
DOI: https://doi.org/10.11144/Javeriana.ie23.frcs

Recibido: 09 Febrero 2021

Aceptado: 14 Octubre 2021

Publicado: 30 Diciembre 2021

\section{Resumen:}

Introducción. El sexting se define como el envío de contenido erótico o pornográfico a través de dispositivos digitales, comúnmente el móvil o smartphone, como una expresión de sexualidad cada vez más frecuente, y a pesar de que este fenómeno puede tener lugar con efectos positivos en quienes lo practican, existe muy poca evidencia relacionada con los mecanismos de participación, los motivos que conllevan, la percepción de las consecuencias, entre otros. Algunos autores lo han relacionado con factores como el consumo de sustancias, la promiscuidad y el nivel socioeconómico. Método. Estudio observacional, transversal y relacional, que incluyó a 300 estudiantes universitarios de enfermería, a través de un muestreo no probabilístico a conveniencia, en el que se aplicaron dos instrumentos, la escala de conductas sobre sexting y el instrumento de nivel socioeconómico familiar NSE AMAI. Resultados. El 64,7 \% declara haber participado en prácticas de sexting, de los cuales el 26,2 \% publicó una imagen suya a través de sus redes sociales, el 13,9\% declaró realizarlo cuando bebe alcohol y el 43,8 \% reportó que es falso que el sexting los hace sentir inmorales. Se encontraron relaciones de prevalencia e intensidad del sexting con el nivel socioeconómico y la vida sexual activa. Conclusión. Al entender los distintos factores que predominan en esta práctica se pueden desarrollar diferentes intervenciones contextualizadas en los grupos poblacionales de riesgo, que sean accesibles por ambas partes, lo que permite una libre expresión de la sexualidad sin comprometer la seguridad de las personas.

Palabras clave: sexting, estudiantes de enfermería, sexualidad, salud pública.

\section{Abstract:}

Introduction: Sexting is defined as sending erotic or pornographic contents through digital devices, usually cell phone or smartphone, which is becoming an increasingly frequent sexual expression. In spite of the fact that it may occur with positive effects for those who do it, there is scarce evidence related to the participation mechanism, the reason driving them, the perception of consequences, among others. Some authors have related sexting to substances use, promiscuity and socioeconomic level. Methods: It is a relational, cross-sectional and observational study including 300 college students of nursing through a non-probabilistic sampling using two instruments, the sexting behavioral scale and the family income-level instrument NSE AMAI. Results: Among the students, $64.7 \%$ stated to have participated in sexting, $26.2 \%$ stated to have posted his/her picture in the social media, $13.9 \%$ stated to do sexting while been drunk and, $43,8 \%$ deemed as false that sexting would make them feel immoral. Prevalence and Notas de autor

\footnotetext{
a Autor de correspondencia: marco.morales@correo.uady.mx
} 
intensity relationship were found between sexting and income level and having an active sexual life. Conclusion: Understanding the different factors prevailing in the sexting allows developing different contextualized interventions in the risk populations groups that can be accessible to both ends. This would allow the free expression of one's sexuality without endangering the people's safety. Keywords: sexting, nursing students, sexuality, public health.

\section{Resumo:}

Introdução. O sexting é definido como o envio de conteúdos eróticos ou pornográficos através de dispositivos digitais, normalmente celulares ou smartphones, como uma expressão cada vez mais frequente da sexualidade e, apesar de este fenómeno ocorrer com efeitos positivos na pratica, há muito pouca evidência relacionada com os mecanismos de participação, os motivos que acarretam, a percepção das consequências, entre outros. Alguns autores o relacionam a fatores como uso de sustâncias, promiscuidade e nível socioeconómico. Método. Estudo observacional, transversal e relacional que incluiu 300 discentes universitários de enfermagem, através de amostragem não probabilística a conveniência, no que foram aplicados dois instrumentos, a escala de conduta sobre sexting e o instrumento de nível socioeconómico familiar NSE AMAI. Resultados. 64,7\% declararam ter participado em práticas de sexting, dos quais 26,2\% publicaram uma imagem sua através das suas redes sociais, 13,9\% declararam fazê-lo quando ingerem álcool e 43,8\% relataram que é falso que o sexting os faça sentir imorais. Encontraram-se relações de prevalência e intensidade do sexting com o nível socioeconómico e a vida sexual ativa. Conclusão. Ao compreender os diferentes fatores que predominam nesta prática podem se desenvolver diferentes intervenções contextualizadas nos grupos populacionais de risco acessíveis por ambas as partes, o que permite uma livre expressão da sexualidade sem comprometer a segurança das pessoas.

Palavras-chave: sexting, discentes de enfermagem, sexualidade, saúde pública.

\section{Introducción}

La sexualidad es una dimensión central del ser humano que está presente en todas las etapas de la vida. El disfrute pleno de la sexualidad y el placer son fundamentales para la salud y el bienestar físico, mental y social (1).

Dentro de este gran concepto, el erotismo, es decir, la expresión sensual, versa sobre los sentidos y su satisfacción, la capacidad para sentir deseo, excitación, orgasmo y placer. Abarca las caricias, las fantasías y la imaginación que evocan internamente aquellos recuerdos o imágenes que desencadenan la excitación sexual (2).

Actualmente, con la llegada y posicionamiento del internet como principal medio para obtener y seleccionar información, son importantes las habilidades de manejo y comunicación, ya que, gracias a ello, podemos establecer contactos más rápido que nunca $(3,4)$.

Es así que estas plataformas se han vuelto importantes en los procesos de socialización, y se reconoce que este progreso tecnológico puede traer ventajas tales como la eliminación del tiempo de espera entre respuestas, la creación de contenido único para representar ideas, el uso de material audiovisual para comunicar emociones, entre otros, incluso el aumento de la intimidad, para expresar ideas y emociones a distancia en tiempo real; por otro lado, algunas desventajas se refieren a la despersonalización, es decir, al traslado del afecto y las atenciones al dispositivo celular, la ansiedad por el tiempo de respuesta entre las personas y el almacenamiento de contenido sin el consentimiento de los involucrados (5).

Dentro de los fenómenos que las parejas pueden realizar en la comunicación digital se encuentra el sexting, que es definido como el envío de mensajes y contenido audiovisual sexual o pornográfico a través de dispositivos electrónicos. Existen dos vertientes de estudio acerca de la percepción teórica del sexting: una lo entiende como una práctica íntima y naturalizada en la era digital, y otra lo postula como un comportamiento desviado y arriesgado (6).

Así, algunos estudios sugieren que este comportamiento es una diversión que se usa principalmente para comenzar o mantener una relación íntima, mientras que otros, con evidencia creciente, apoyan la perspectiva desviada, vinculando el sexting con complejas consecuencias negativas (7).

En cuanto a la prevalencia de su realización, se tienen muy pocos estudios cuyas muestras sean lo suficientemente representativas para poder hacer inferencias con poder estadístico. Dentro de ellos, el estudio 
de los Estados Unidos en 2008 y 2009 de Amanda Lenhardt para Pew Internet incluyó 800 adolescentes y determinó que un $4 \%$ de los jóvenes de entre 12 y 17 años había enviado un mensaje con contenido sexual y el $15 \%$ los había recibido (8). Por otro lado, entre jóvenes adultos (de 18 a 29 años) un metaanálisis publicado sugiere una participación de hasta 38,3 \% en el envío de este tipo de mensajes y 41,5 \% en su recepción, de una muestra con 18122 participantes, entre 50 estudios heterogéneos (9).

Esta prevalencia aparece mediada por diversos factores, como la edad y el sexo, y aspectos étnico-sociales como el nivel socioeconómico (por el acceso al internet, así como la mayor utilización de dispositivos digitales en estratos más altos), la educación sexual y la percepción de las consecuencias, ya que para quienes practican el sexting y perciben consecuencias positivas es mayor la probabilidad de repetición, mientras que quienes lo sienten como una conducta desviada tienden a evitarlo (10).

Agregado a esto, diversos estudios han relacionado esta práctica con conductas que pueden ser perjudiciales para la salud, tales como el uso de alcohol (3,78 Odds Ratio $[\mathrm{OR}])$ previo y durante el sexting, el consumo de tabaco (2,66 OR) y otras drogas (3,48 OR) (11).

Respecto a la percepción de las consecuencias, es importante abordar la dicotomía frente al estudio del sexting. Si bien es establecido en la Asamblea General de la Asociación Mundial para la salud sexual que las personas tienen derecho a vivir conforme a la propia expresión sexual e intimidad (12), con consentimiento, y diversos estudios remarcan la gratificación en cuanto al aumento de la excitación sexual, intimidad y reducción de las inhibiciones (13), por otro lado, los participantes pueden percibir que el sexting puede herir elementos tales como su reputación, carrera, autoestima y su estatus actual en la relación (14).

A través de investigaciones independientes, en España se ha encontrado que los estudiantes de enfermería utilizaron las redes sociales para "ligar" o realizar encuentros de carácter romántico, y un 60 \% había recibido imágenes con contenido erótico. La carrera de Enfermería, al ser integrada en su mayoría por mujeres puede presentar una vulnerabilidad agregada al estudiante universitario común, y además se resalta su papel en la promoción de la salud en los contextos escolares y comunitarios, por lo que el conocimiento de este tema resulta de vital importancia para la salud pública (15).

$\mathrm{Al}$ estar en constante aislamiento físico y social, y presentar una intensa carga emocional, debido a la combinación de prácticas y clases, los estudiantes de enfermería pueden entrar en apego con los dispositivos celulares para sentirse en constante conexión con diferentes personas, por lo que la expresión afectiva a través del celular se vuelve un fenómeno cotidiano en su medio, haciéndolos vulnerables a las consecuencias negativas de estas prácticas (16).

Es por ello, que para poder disfrutar libremente de los beneficios que puede traer esta práctica y disminuir la probabilidad de eventos negativos, los estudios deben no solo caracterizar las particularidades tecnológicas sobre la realización del sexting, sino apuntar a entender las conductas relacionadas (caracterización de las emociones, conductas y motivación durante la práctica) y los aspectos que pueden focalizar las campañas de información, en los grupos etarios deseados (17).

Ante esto, se plantean los objetivos de caracterización de las conductas sobre sexting y descripción de los factores asociados con su prevalencia en un grupo de estudiantes universitarios de enfermería.

\section{Materiales y métodos}

Estudio de tipo observacional, de corte transversal y alcance correlacional. Se utilizó un muestreo no probabilístico a conveniencia, en la división académica de Enfermería de una universidad pública. Para ello, se solicitó el permiso de las autoridades institucionales y se les presentó a los estudiantes el objetivo de esta investigación, así como el mecanismo de su participación (el llenado de la batería de los instrumentos). Posteriormente, en grupos reducidos, se aplicó con los estudiantes que deseaban participar. La batería estuvo constituida por 3 secciones principales: 
- Datos sociodemográficos y de identificación, que abarca reactivos respecto a la edad, sexo, año cursado de carrera, lengua indígena, situación de pareja, y consumo de bebidas alcohólicas, tabaco y otras drogas (marihuana, metanfetaminas, inhalables u otros).

- Encuesta de Nivel Socioeconómico Familiar NSE AMAI 2016, utilizada por el Instituto Nacional de Estadística y Geografía para poder estratificar el nivel socioeconómico familiar. Esta posee un alfa de Cronbach de 0,944 y se compone de seis preguntas de opción múltiple, para lo cual cada opción de respuesta devuelve un puntaje, cuya suma total se agrupa en siete estratos (A/B, C+, C, C-, D+, D, E) (18).

- Escala de Conductas Sobre Sexting (Sexting Behaviors Scale), compuesta por 28 preguntas con valores que van del 0 al 4, y que de acuerdo con la naturaleza de la pregunta permite caracterizar tres ámbitos: disposición hacia la participación en el sexting, participación real y expresión emocional (Alfa de Cronbach $=0,922)$. La sumatoria del instrumento se clasifica en cinco niveles de intensidad de participación: muy bajo (0 a 27), bajo (28 a 55), medio (56 a 83), alto (84 a 111) y muy alto (112 a 135) (17).

Para la descarga y el análisis de la información se utilizó el programa SPSS V.21 que permite obtener medidas de tendencia central y dispersión, así como las pruebas de comparación de medias para grupos (U de Mann Whitney y correlación de Pearson). Posteriormente, se utilizó el programa Microsoft Excel para la tabulación de los datos, así como para su presentación en gráficas. Finalmente, el programa EpiDat 3.0 se utilizó para medir los OR en los factores asociados. Es importante aclarar que los resultados de esta investigación fueron devueltos mediante un informe a la dependencia correspondiente, donde pueden ser consultados por todas y todos los interesados.

Finalmente, es importante mencionar que este proyecto fue aprobado por el Comité de Ética e Investigación de la Unidad Académica Institucional correspondiente (folio: UAM-CEI-01-19). Esta investigación se clasifica como de riesgo mínimo, según las normativas nacionales vigentes, y respeta los principios bioéticos propuestos en la declaración de Helsinki, tales como la protección de la privacidad y el anonimato de los participantes, la entrega y firma del consentimiento informado, garantizándose que los beneficios derivados de los datos utilizados superan los riesgos (19).

\section{Resultados}

De los 300 participantes, el 25,7 \% (77) son hombres y el 74,3\% (223) son mujeres, con una media de edad fue de 20,7 años (DE 1,9); además, el 53,7 \% (161) se encontraba en el cuarto año de la carrera, y el 49,3 $\%$ (148) estaba en una relación. En cuanto a la etnicidad, se incluyeron dos preguntas de identificación de la comunidad indígena relacionadas con la lengua (si la persona o alguien de su familia lo hablaba), obteniendo un 6,3\% (19) de identificación con este grupo. Por otro lado, se les preguntó acerca del consumo actual de sustancias (alcohol, tabaco y otras drogas), obteniendo respuestas afirmativas hasta en un 87 \% (261). La totalidad de frecuencias y porcentajes de los datos sociodemográficos se presentan en la tabla 1. 
TABLA 1

Datos sociodemográficos de los participantes

\begin{tabular}{|c|c|c|}
\hline Variable & Porcentaje & Frecuencia \\
\hline \multicolumn{3}{|l|}{ Sexo } \\
\hline Hombre & $25,7 \%$ & 77 \\
\hline Mujer & $74,3 \%$ & 223 \\
\hline \multicolumn{3}{|l|}{ Conexión a internet (distinta a móvil o datos) } \\
\hline $\mathrm{Si}_{1}$ & $87,3 \%$ & 262 \\
\hline No & $12,7 \%$ & 38 \\
\hline \multicolumn{3}{|l|}{ Año escolar que se encuentra cursando } \\
\hline Primero & $30 \%$ & 90 \\
\hline Segundo & $15,7 \%$ & 47 \\
\hline Tercero & $0,6 \%$ & 2 \\
\hline Cuarto & $53,7 \%$ & 161 \\
\hline \multicolumn{3}{|l|}{ Condición indigena } \\
\hline 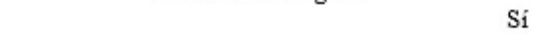 & $6,3 \%$ & 19 \\
\hline No & $93,7 \%$ & 281 \\
\hline \multicolumn{3}{|l|}{ Pareja } \\
\hline Si & $49,3 \%$ & 148 \\
\hline No & $51,7 \%$ & 152 \\
\hline \multicolumn{3}{|l|}{ Consumo de alcohol } \\
\hline (1) & $87 \%$ & 261 \\
\hline No & $13 \%$ & 39 \\
\hline \multicolumn{3}{|l|}{ Consumo de tabaco } \\
\hline $\mathrm{Si}$ & $45 \%$ & 135 \\
\hline No & $55 \%$ & 165 \\
\hline \multicolumn{3}{|l|}{ Consumo de otras drogas } \\
\hline (1) & $20,3 \%$ & 61 \\
\hline No & $79,7 \%$ & 239 \\
\hline \multicolumn{3}{|l|}{$\begin{array}{c}\text { Habia recibido educación sexual antes de los } 18 \\
\text { años }\end{array}$} \\
\hline 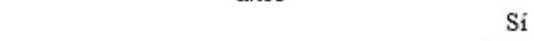 & $97,3 \%$ & 292 \\
\hline No & $3,7 \%$ & 8 \\
\hline \multicolumn{3}{|l|}{ Habia tenido relaciones sexuales } \\
\hline 皮 & $78 \%$ & 234 \\
\hline No & $22 \%$ & 66 \\
\hline
\end{tabular}

Fuente: Elaboración propia, $n=300$

En la medición del nivel socioeconómico familiar, mediante los resultados del instrumento NSE AMAI, la mayor parte de los estudiantes se encontraban en el nivel C+ con un 34,3\% (103), seguido por A/B con $25 \%$ (75), C con $24 \%$ (72), entre otros. El promedio general de los sujetos fue de 176,4 (DE 44,1), es decir, de acuerdo con la escala se sitúa en el nivel C+ (alto bajo).

Respecto a la educación sexual, se les preguntó si habían recibido educación sexual antes de los 18 años, a lo que 292 estudiantes (97,3\%) respondieron que sí la habían recibido. Al analizar esta variable por sexo, se obtiene que el 96,1 \% de los hombres (74) declara haberla recibido, frente al $100 \%$ de las mujeres. En cuanto a la vida sexual activa, se obtiene una edad promedio de inicio de 17,5 años (2,02 DE), con una mínima de 12 años y una máxima de 23. En promedio, un 81,8 \% (63) de hombres había iniciado su vida sexual con 16,5 años (2,0 DE), mientras que un 76,6\% (171) de mujeres manifestaron una edad promedio de inicio de 17,83 años $(2,2 \mathrm{DE})$.

Al cuestionarles acerca de si había realizado sexting, los participantes declararon en un $64,7 \%$ (194) haberlo hecho. Se describe la prevalencia de estudiantes que declara haber participado en este intercambio de contenido en la figura 1 . 


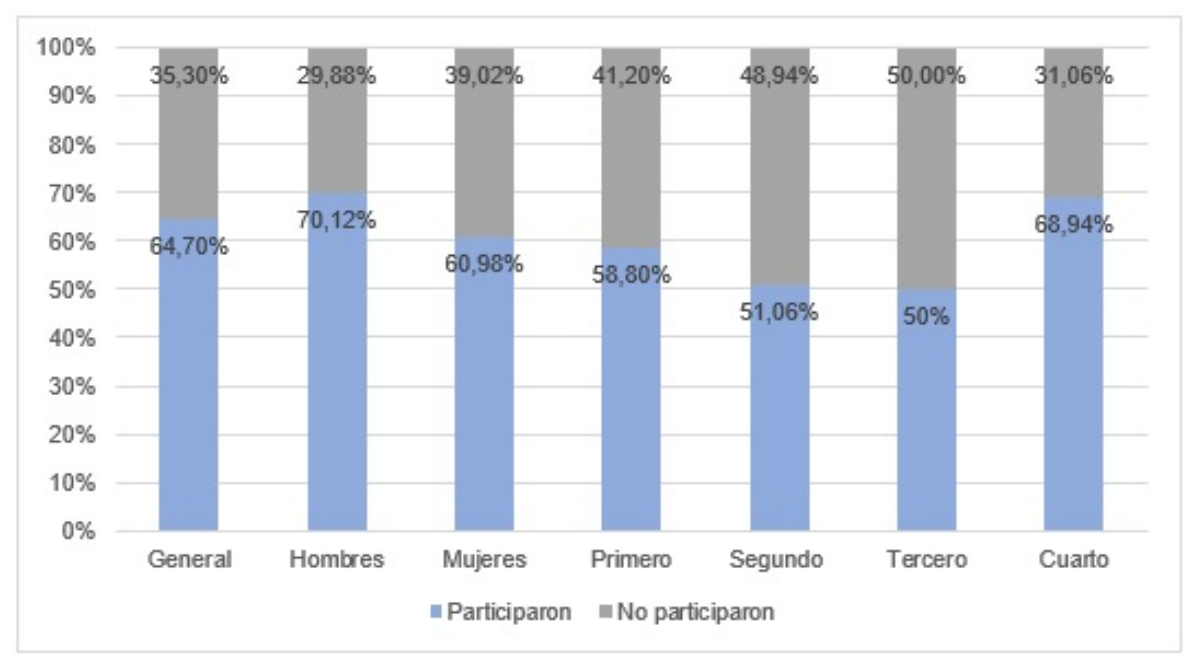

FIGURA 1

Porcentaje de participación en sexting, general y por grupos

Fuente: Elaboración propia, $\mathrm{n}=194$

Posteriormente, a través del instrumento de indagación de conductas sobre sexting, se describe el comportamiento de los participantes que declararon haberlo hecho, estableciendo que el 90,72\% había recibido imágenes con contenido sexual y el 92,26 \% lo había practicado en forma de mensaje de texto. En cuanto a las plataformas, el 75,7 \% (147) recibió imágenes o mensajes provocativos insinuantes en plataformas como Facebook, WhatsApp, Instagram o Snapchat, y el 55,6 \% (108) los respondió. Finalmente, el 26,2 \% (51) declaró haber compartido en modo público imágenes insinuantes o provocativas en plataformas públicas como Facebook, Instagram, Tumblr o Snapchat.

Al preguntar sobre los hábitos correspondientes a la participación real en el sexting, el 63,9\% (124) de los participantes que declararon hacer sexting, reportaron hacerlo con su pareja, y el 29,8 \% (58) determinó hacerlo con alguien que les atrae. Posteriormente, cuando se les preguntó si lo hacen con amigos, el 14,9\% (29) respondió afirmativamente. También se encontró que el 9,7 \% (19) realiza sexting cuando está de fiesta con amigos, un 13,9\% (27) lo hace cuando bebe alcohol y el 3,6 \% (7) cuando está fumando marihuana o consumiendo otras drogas. En la figura 2 se describen las respuestas a la pregunta sobre los motivos de realización. 


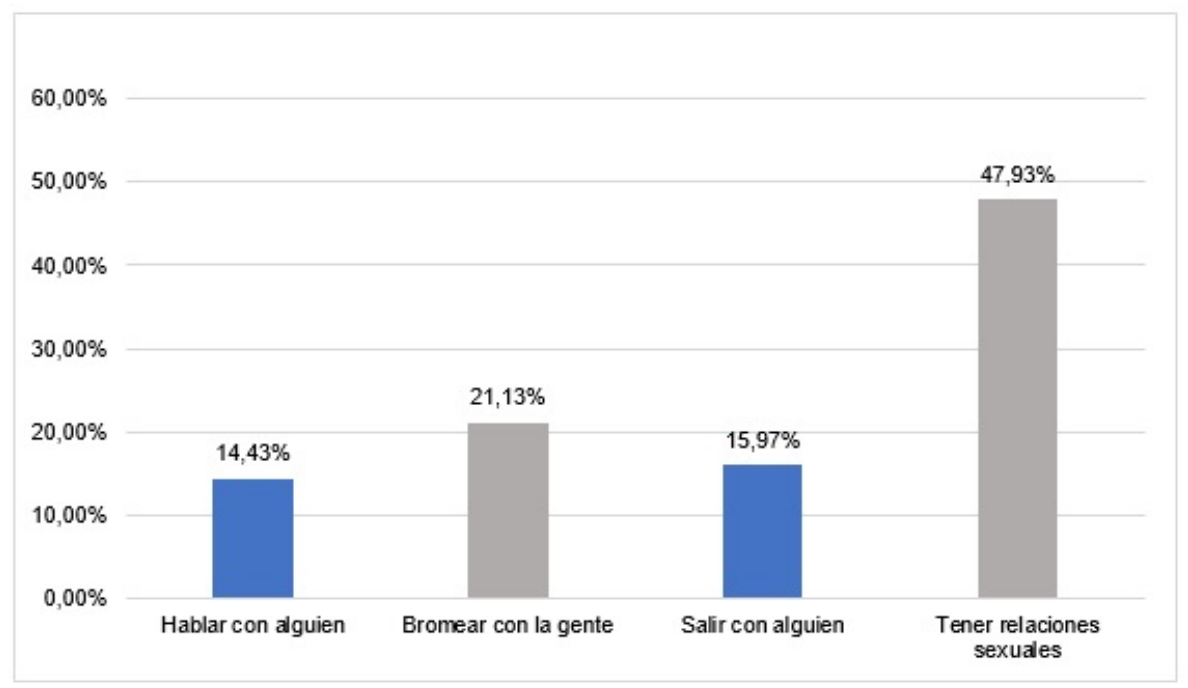

FIGURA 2

Motivos de participación para hacer sexting

Fuente: Elaboración propia, $\mathrm{n}=194$

En el último apartado de este instrumento, la expresión emocional durante el sexting, para la primera pregunta ¿el sexting hace que tenga más probabilidad de tener sexo o salir con alguien?, los participantes respondieron afirmativamente en un 49,4\% (96). Por su parte, el 43,8 \% (85) reportó que es falso que el sexting lo haga sentir inmoral, mientras que el 57,7 \% (112) declara sentirse avergonzado y el $48.9 \%$ (95) feliz mientras lo hacen. En la tabla 2 se pueden visualizar los porcentajes de participación, de acuerdo con los factores sociodemográficos evaluados.

TABLA 2

Distribución del porcentaje de participación en sexting por variable sociodemográfica y prueba de relación con OR.

\begin{tabular}{|c|c|c|c|c|}
\hline \multicolumn{3}{|c|}{ Identificación con la variable de los practicantes de sexting } & \multicolumn{2}{|c|}{ Relación con $O R$} \\
\hline & Si & No & Valor OR & $\begin{array}{c}\text { Intervalo de } \\
\text { confianza }\end{array}$ \\
\hline $\begin{array}{r}\text { Conexión a internet (distinta a móvil o } \\
\text { datos) }\end{array}$ & $88,7 \%(172)$ & $11,3 \%(22)$ & 1,38 & $0,96-2,778$ \\
\hline Condición indigena & $27,16 \%(14)$ & $72,84 \%(180)$ & 0,65 & $0,17-2,49$ \\
\hline Pareja & $54,1 \%(105)$ & $45,9 \%(89)$ & 1,72 & $1,06-2,79$ \\
\hline Consumo de alcohol & $88,7 \%(172)$ & $11,3 \%(22)$ & 1,49 & $0,76-2,93$ \\
\hline Consumo de tabaco & $53,6 \%(104)$ & $46,4 \%(90)$ & 2,79 & $1,68-4,63$ \\
\hline Consumo de otras drogas & $25,3 \%(49)$ & $74,7 \%(145)$ & 2,64 & $1,337-5,23$ \\
\hline Había recibido educación sexual antes de & $96,4 \%(187)$ & $3,6 \%(7)$ & 0,25 & $0,03-2,09$ \\
\hline \multicolumn{5}{|l|}{ los 18 años } \\
\hline Habia tenido relaciones sexuales & $87,1 \%(169)$ & $12,9 \%(25)$ & 4,26 & $2,40-7,56^{*}$ \\
\hline \multicolumn{5}{|c|}{ Nivel socioeconómico } \\
\hline Alto alto $(\mathrm{A} / \mathrm{B})$ & $26,3 \%(51)$ & $73,7 \%(143)$ & & \\
\hline Alto bajo $(\mathrm{C}+)$ & $35,6 \%(69)$ & $64,6 \%(125)$ & & \\
\hline Medio alto (C) & $21,1 \%(41)$ & $78,9 \%(153)$ & & \\
\hline Medio bajo (C-) & $11,3 \%(22)$ & $88,7 \%(172)$ & & \\
\hline Bajo alto (D+) & $4,1 \%(8)$ & $95,9 \%(186)$ & & \\
\hline Bajo bajo (D) & $1,5 \%(3)$ & $98,5 \%(191)$ & & \\
\hline
\end{tabular}

Fuente: Elaboración propia, $n=194$

Es así como, a través de la escala previamente descrita, se puede identificar que los sujetos realizan mayoritariamente el sexting con una intensidad baja $(68,8 \%, 122)$, mientras que para intensidades medias y altas se reportan totales de $35 \%(68)$ y $1 \%(2)$, respectivamente.

De acuerdo con el sexo, los hombres tienen un nivel de intensidad de participación promedio de 37,7 (DE 27,1) y las mujeres de 31,9 (DE 26,3), lo cual ubica a ambos grupos en un nivel de intensidad bajo (gráfica 3). Para determinar si los grupos presentaban diferencias estadísticamente significativas, se realizó la prueba $U$ de Mann Whitney en la variable sexo (Hipótesis nula=no existen diferencias estadísticamente significativas, $\mathrm{p}<0,05$ ), con la cual se obtuvo un valor de 0,746 , determinando que no existen efectivamente diferencias significativas en las medianas de participación. 
Finalmente, después de comprobar la normalidad de las variables con la prueba de Shapiro-Wilk $(\mathrm{p}<0,001)$, se correlacionó el puntaje establecido en el instrumento NSE AMAI con la puntuación total obtenida en la escala de conductas sobre sexting, mediante la prueba de correlación de Pearson $(\mathrm{p}<0,05)$, obteniendo un valor de correlación de 0,161 , con una significancia bilateral de 0,025 .

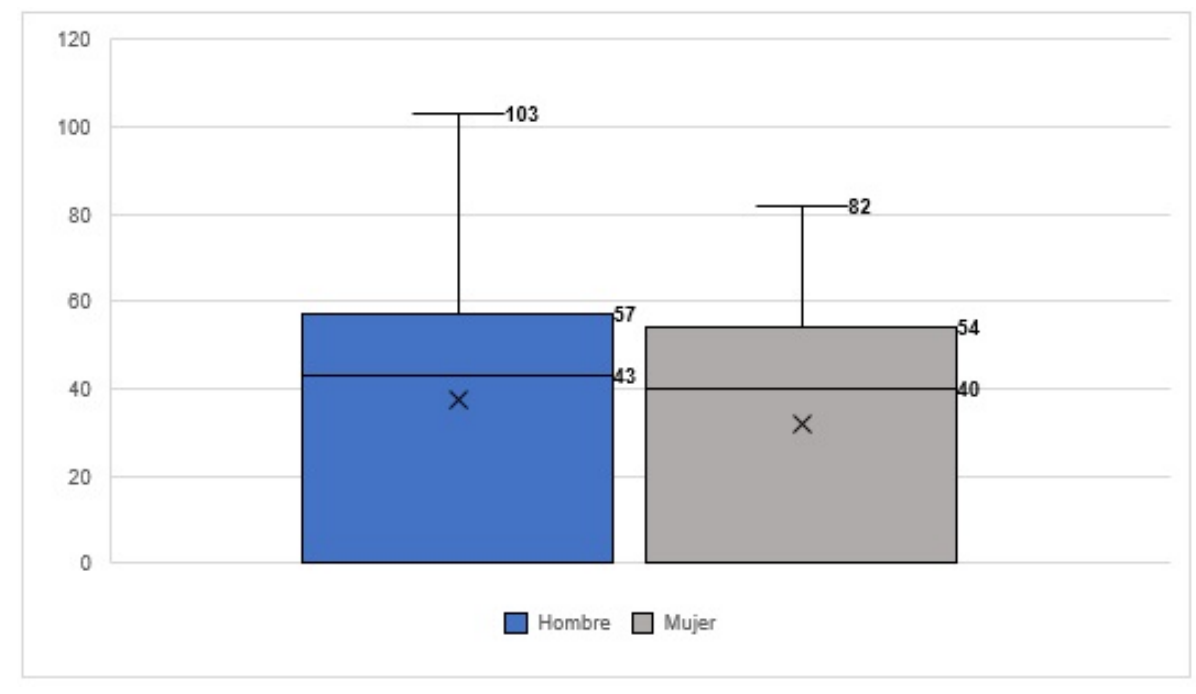

FIGURA 3

Intensidad de participación por sexo.

Fuente: Elaboración propia, $\mathrm{n}=194$

\section{Discusión}

Es innegable que la comunicación en el contexto actual ha dado pasos agigantados en los últimos años, y que la usabilidad, transportabilidad y las diversas herramientas y plataformas en los celulares han provocado que se entre en una era de comunicación digital como nunca se había observado. Por lo tanto, el deber y quehacer del salubrista debe de enfocarse en la prevención de los eventos riesgosos o negativos que puedan derivar de ciertas prácticas, y a la promoción de una sexualidad positiva y sana.

El estudio de las conductas sobre sexting permite caracterizar el fenómeno no solo en sus factores tecnológicos, sino entender realmente los motivos de su realización, el contexto en el cual se hace y la percepción que se tiene sobre ello y sus consecuencias.

De acuerdo con los objetivos de esta investigación, se establecieron las medidas descriptivas relacionadas con la población y la caracterización del sexting, posibilitando un análisis de resultados que indica que el 64,7 \% declaró haber realizado esta práctica, lo cual indica una alta prevalencia, ya que en investigaciones reportadas por The Associated Press \& MTV en Estados Unidos se determinó un total de $33 \%$ de un grupo de 18 a 24 años que sí había realizado sexting, mientras que otros estudios con estudiantes de enfermería determinaron una prevalencia de participación de $63 \%$ (15) (20).

Asimismo, se observa que, en las parejas reportadas, el flujo de mensajes con contenido sexual fue alto, ya que en este estudio fue de $90,72 \%$ para imágenes y $92,26 \%$ para texto, mientras que en (21) se reporta como de $14,3 \%$. Esto parece apuntar a que el sexting inicia y prevalece más como mensajes de texto, y posteriormente pasa a practicarse con imágenes. En el mismo estudio mencionado se reportó una prevalencia de $11,3 \%$ de sexting entre amigos, mientras que en el actual estudio fue de 29,8 \%, y de 63,9\% con la pareja, con lo cual podemos interpretar que la mayor parte de estas prácticas se realizan entre parejas de adultos jóvenes.

Las motivaciones son uno de los principales factores al momento de estudiar las conductas sobre sexting, ya que podrían ser la pieza clave para incidir sobre los elementos conductuales del problema y dotar a los 
participantes de mejores herramientas de manejo digital. En este estudio, un 14,4\% de los participantes respondió que su motivación para hacer sexting es conocer a alguien, mientras que un 15,9\% lo hace cuando quiere comenzar a salir con alguien y la gran mayoría (47,9\%), cuando quiere tener relaciones sexuales. Estos porcentajes difieren con lo reportado en (22), donde se afirma que, de los 228 participantes, el $34 \%$ realiza prácticas de sexting para conocer o coquetear con alguien, el $44 \%$ respondió que lo hacen para asegurarse una relación romántica exclusiva, y el $33 \%$ con el objetivo de tener encuentros sexuales en un futuro cercano. Hasta este punto, podemos determinar que el sexting, entre adultos, se realiza principalmente con la pareja, con la intención de tener relaciones sexuales.

En el último apartado de este instrumento se evalúa la expresión emocional durante y después del sexting, respecto de lo cual el 43,8\% reporta que es falso que el sexting lo hace sentir inmoral, el 48,9\% afirma sentirse feliz mientras lo hace, y el 57,7 \% reconoce sentir algo de vergüenza al realizarlo. Esto es importante, ya que establece elementos sobre la percepción del riesgo que tienen los participantes, pudiéndose establecer para este grupo que la percepción de las consecuencias negativas es baja, lo cual concuerda con lo reportado en (23), sobre un grupo de 1839 participantes donde el $7 \%$ de ellos aseguró que se sentían avergonzados por realizar sexting y creían que podría dañar su reputación (24).

En cuanto a las asociaciones, en este estudio se encontró una relación positiva, medida a través de Odds Ratio, entre esta práctica y el tener relaciones sexuales, lo cual concuerda con la principal motivación reportada, y a través de la prueba de correlación de Spearman con el nivel sociodemográfico familiar. Esto difiere con lo anteriormente reportado por (11), quienes encontraron asociaciones positivas con el uso de alcohol (3,78 OR), tabaco (2,66 OR) y otras drogas (3,48 OR), por lo cual no se asocia algún comportamiento de riesgo de consumo de sustancias. Por otro lado, el nivel socioeconómico puede ser explicado por la relación entre esa misma variable y el acceso y uso de las tecnologías.

Dentro de las fortalezas y aportes a la práctica, se destaca la posibilidad de utilizar la información recogida y analizada para implementar campañas de sexualidad positiva que prevengan efectos negativos. Pueden plantearse intervenciones como la promoción del uso de aplicaciones y plataformas capaces de encriptar los datos en conversaciones específicas (para los que realizan sexting en pareja), que inhabilitan las funciones de guardar imágenes y tomar capturas de pantalla, así como borran automáticamente las conversaciones después de un límite de tiempo, entre otras funciones, por lo que un enfoque hacia la sexualidad positiva podría proporcionar las herramientas necesarias a los usuarios para poder gozar plenamente de sus beneficios y minimizar los riesgos.

Dentro de las limitaciones, se declara que, al ser un análisis no probabilístico, esto puede afectar en su representatividad y poder estadístico, por lo que se recomienda ampliar la población de estudio.

\section{Conclusión}

La salud pública y la enfermería convergen en la ciencia y arte de impedir enfermedades, prolongar la vida, fomentando la salud y el bienestar a través del esfuerzo organizado con la comunidad, y estas acciones deben estar enfocadas para que cada ciudadano se encuentre en condiciones de disfrutar de sus beneficios en salud física, mental y emocional.

La expresión de la sexualidad a través del sexting es una práctica que no puede ser negada, tratar de prevenirla con educación sexual basada en un modelo de riesgo es un factor que puede llevar a consecuencias como el cyberbulling y la pornovenganza, por lo que varios autores proponen reconceptualizar el enfoque hacia un entorno de empoderamiento, en el cual se proporcionen las herramientas necesarias a los individuos para que ejerzan su sexualidad de forma segura.

De este modo, en consecuencia con el movimiento sex-affirmative se determina que una perspectiva empoderadora, enmarcada en el consentimiento de ambas partes, puede ofrecer una educación innovadora 
y acorde con los deseos y valores de quienes realizan estas prácticas, es decir que se trata de dejar de centrar las campañas en mensajes orientados hacia la prohibición y el miedo para redireccionarlas hacia un sexting seguro y con consentimiento, de forma que las personas puedan disfrutar con la pareja deseada los beneficios de esta práctica.

\section{Bibliografía}

1. Instituto de Seguridad y Servicios Sociales de los Trabajadores del Estado. Cartilla de derechos sexuales de adolscentes y jóvenes. Informe. México: ISSSTE, Prensa; 2018.

2. Howard D, Klettke B, Ling M. Does body dissatisfaction influence sexting behaviors in daily life? Computers in Human Behavior. 2019;101(10): 320-26. https://doi.org/10.1016/j.chb.2019.07.033

3. Davis M, Powell A, Gordon D. I want your sext: sexting and sexual risk in emerging adult minority men. AIDS Education and Prevention. 2016; 28(2): 138-52. https://doi.org/10.1521/aeap.2016.28.2.138

4. Widman L, Javidi H, Maheux A. Sexual Communication in the digital age: sdolescent sexual communication with parents and friends about sexting, pornography, and starting relationships online. Sexuality \& Culture. 2021; 25(2): 1-18. DOI:https://doi.org/10.1007/S12119-021-09866-1

5. Van Oosten J, Vandenbosch L. Sexy online self-presentation on social network sites and the willingness to engage in sexting: a comparison of gender and age. Journal of Adolescence. 2017; 54(1): 42-50. https://doi.org/10.10 16/j.adolescence.2016.11.006

6. Barrense Y, Berchtold A, Surís J, Ajre C. Sexting and the definition Issue. Journal of Adolescent Health. 2017; 31(1). DOI: https://doi.org/10.1016/j.jadohealth.2017.05.009

7. Broaddus M, Dickson J. The uses of texting in sexual relationships scale: asoociations with risky sexual behavior among at-risk african american emerging adults. AIDS Education and Prevention. 2016; 28(5): 393-404. DOI: https://doi.org/10.1521/aeap.2016.28.5.393

8. Albury K, Crawford K. Sexting, consent and young people's ethics: Beyond Megan's Story. Journal of media \& cultural studies. 2012; 26(3): 463-73. https://doi.org/10.1080/10304312.2012.665840

9. Mori C, Cooke J, Temple J, Ly A, Lu Y, Anderson N, et al. The prevalence of sexting behaviors among emerging adults: a meta-analysis. Arch Sex Behav. 2020; 49(4): 1109-19.

10. Bauermeister J, Yeagley E, Meanley S, Pingel E. Sexting among young men who have sex with men: results from a national survey. Journal of Adolescent Health. 2014; 54(5): 606-11. https://doi.org/10.1016/j.jadohealth.20 13.10.013

11. Benotsch E, Snipes D, Martin A, Bull S. Sexting, substance use, and sexual risk behavior in young adults. Journal of Adolescent Health. 2015; 20(1): 307-13. doi: https://doi.org/10.1016/j.jadohealth.2012.06.011

12. López M. Derechos sexuales y reproductivos: un asunto de derechos humanos. Informe. Mexico: CNDH, Programa Especial de VIH/Sida y Derechos Humanos; 2017.

13. Naezer M. From risky behaviour to sexy adventures: reconceptualising young people's online sexual activities. Culture, Health \& Sexuality. 2018; 20(6):715-29. doi: 10.1080/13691058.2017.1372632

14. García J, Gesselman A, Silman S, Perry B, Coe K, Fischer H. Sexting among singles in the USA: prevalence of sending, receiving, and sharing sexual messages and images. Sexual Health. 2016; 13(1): 428-35. DOI: https:/ /doi.org/10.1071/SH15240

15. Gutiérrez-Puertas V, Gutiérrez-Puertas L, Aguilera-Manrique G, Baños-Martín MDM, Granados-Gámez G, Márquez-Hernández VV. The sexting phenomenon in spanish nursing students. Computers, Informatics, Nursing. 2017; 35(8): 425-30. doi:https://doi.org/10.1097/CIN.0000000000000334

16. Cho S, Lee E. Development of a brief instrument to measure smartohone addiction among nursing students. CIN: Computers, Informatics, Nursing. 2015; 33(5). DOI: https://doi.org/10.1097/CIN.0000000000000132 
17. Chacón-López H, Romero-Barriga JF, Aragón-Carretero Y, Caurcel-Cara M. . Construcción y validación de la escala de conductas sobre sexting. REOP. 2016; 27(2): 99-115. https://doi.org/10.5944/reop.vol.27.num.2.20 16.17116

18. Comité de Nivel Socioeconómico AMAI. Nivel Socio Económico AMAI 2018. Nota Metodológica. Asociación Mexicana de Agencias de Inteligencia de Mercado y Opinión; 2017.

19. Cámara de diputados del H. Congreso de la Unión. Ley General de Salud. Última Reforma DOF 12-07-2015; 2015.

20. A Thin Line. Executive summary: 2011 AP-MTV Digital Abuse Study. [Online]; 2011 [cited 2020 Enero 06. Disponible en: https://on.mtv.com/38snuoP.

21. Patchin J, Hinduja $S$. The nature and extent of sexting among a national sample of middle and high school students in the U.S. Archives of Sexual Behavior. 2019; 48(8): 2333-43. doi: https://doi.org/10.1007/s10508-019-1449-y

22. Strohmaier H, Murphy M, DeMatteo D. Youth Sexting: prevalence rates, driving motivations, and the deterrent effect of legal consequences. Sex Res Soc Policy. 2014; 11(3): 245-55. https://doi.org/10.1007/s13178-014-0 $162-9$

23. Rice E, Rhoades $\mathrm{H}$, Winertrobe $\mathrm{H}$. Sexually explicit cell phone messaging associated with sexual risk among adolescents. PEDIATRICS. 2012; 130(4): 667-73.

24. Levine D. Sexting: A terrifying health risk. or the New Normal for Young Adults? Journal of Adolescent Health. 2013; 52(1): 257-58.

\section{Notas}

* Artículo original de investigación.

Licencia Creative Commons CC BY 4.0

Financiación: Ninguna.

Conflicto de intereses: Los autores declaran no tener conflictos de intereses.

Cómo citar este artículo: Morales Rojas M. C., Valle Solís M. O., Benítez Guerrero M., López Flores J. F. Factores relacionados y conductas sobre sexting en estudiantes de enfermería en una universidad pública. Investigación en Enfermería, Imagen y Desarrollo. 2021;23. https://doi.org/10.11144/Javeriana.ie23.frcs 